\title{
A non-inferiority comparison of Delta Medical's PEEK Suture Anchor and Smith \& Nephew's PEEK Suture Anchor in arthroscopic rotator cuff repair: a multicenter prospective single- blind randomized controlled clinical trial
}

\author{
Peng Gao ${ }^{1 \#}$, Hongtao Wang ${ }^{1 \#}$, Yongsheng $\mathrm{Xu}^{2}$, Yanlin $\mathrm{Li}^{3}$, Guofeng $\mathrm{Cai}^{3}$, Yufeng $\mathrm{Wu}^{4}, \mathrm{Ziqi}^{\mathrm{i}} \mathrm{Huang}^{4}$, \\ Qiang Li $^{5}$, Jing Wang ${ }^{1}$ \\ ${ }^{1}$ Department of Joint Surgery and Sports Medicine, Hunan Provincial People's Hospital (The First-affiliated Hospital of Hunan Normal University), \\ Changsha, China; ${ }^{2}$ Department of Bone and Joint Surgery, Inner Mongolia Autonomous Region People's Hospital, Huhhot, China; ${ }^{3}$ Department \\ of Sport Medicine, First Affiliated Hospital of Kunming Medical University, Kunming, China; ${ }^{4}$ Department of Orthopedics, Traditional Chinese \\ Medicine Hospital of Zhongshan, Zhongshan, China; ${ }^{5}$ Department of Orthopedics, Traditional Chinese Medicine Hospital of Huaihua (Affiliated \\ Hospital of Traditional Chinese Medicine, Hunan University of Medicine), Huaihua, China \\ Contributions: (I) Conception and design: P Gao, J Wang; (II) Administrative support: Y Xu, J Wang; (III) Provision of study materials or patients: G \\ Cai, Z Huang; (IV) Collection and assembly of data: H Wang, Y Xu, Y Li, Y Wu; (V) Data analysis and interpretation: P Gao, Q Li; (VI) Manuscript \\ writing: All authors; (VII) Final approval of manuscript: All authors. \\ \#These authors contributed equally to this work. \\ Correspondence to: Qiang Li. Department of Orthopedics, Traditional Chinese Medicine Hospital of Huaihua (Affiliated Hospital of Traditional \\ Chinese Medicine, Hunan University of Medicine), Huaihua, China. Email: 18974577077@163.com; Jing Wang. Department of Joint Surgery \\ and Sports Medicine, Hunan Provincial People's Hospital (The First-affiliated Hospital of Hunan Normal University), Changsha, China. \\ Email: wangj0405@163.com.
}

Background: To compare the early clinical outcomes and Magnetic Resonance Imaging (MRI) results of Delta Medical's PEEK (polyether ether ketone) suture anchor with those of Smith \& Nephew's PEEK suture anchor in patients with arthroscopic rotator cuff repair.

Methods: A total of 106 patients in four different medical centers were randomly allocated into two groups: Delta Medical's PEEK suture anchor (53 patients); Smith \& Nephew's PEEK suture anchor (53 patients). The MRI results and early clinical outcomes were evaluated at 3 and 6 months postoperatively. The University of California at Los Angeles (UCLA) shoulder rating scale and the visual analog scale (VAS) score for pain and range of motion were evaluated. MRI were performed at 3 and 6 months postoperatively to examine the integrity of the repaired rotator cuff tendon based on the Sugaya classification.

Results: Significant improvements in pain relief, shoulder function, and functional scores were observed in both groups after surgery regardless of the suture anchor applied $(\mathrm{P}<0.001)$. No differences observed in the functional scores and range of motion. The assessments of UCLA scores at 3 and 6 months produced no statistical differences ( $\mathrm{P}=0.885$ and 0.340 , respectively). The mean VAS scores in group 1 did not reveal statistical differences at 3 and 6 months after surgery compared to group 2. No significant differences in the range of motion were found at each follow-up time-point and no shifting or breakage of the anchors occurred between the two groups $(\mathrm{P}>0.01)$. No major intra- or post-operative complications, such as infection, and vessel or nerve injury.

Conclusions: Pain relief and shoulder function were improved after complete rotator cuff repair in both groups, regardless of the suture anchor applied. The difference in functional scores and range of motion were not significant in groups 1 and 2. Delta Medical's PEEK suture anchor had a non-inferiority effect

\footnotetext{
$\wedge$ ORCID: 0000-0002-4293-7761.
} 
compared to Smith \& Nephew's PEEK suture anchor. Delta Medical's PEEK suture anchor was suitable for arthroscopic rotator cuff repair.

Trial Registration: Chinese Clinical Trial Registry, identifier: ChiCTR2100051716.

Keywords: Arthroscopic rotator cuff repair; polyether ether ketone (PEEK) suture anchor; University of California at Los Angeles (UCLA) scores; visual analog scale (VAS) scores

Submitted Oct 21, 2021. Accepted for publication Dec 08, 2021.

doi: 10.21037/atm-21-6008

View this article at: https://dx.doi.org/10.21037/atm-21-6008

\section{Introduction}

Among all the shoulder pain and disability problems, rotator cuff tear is one of the most important causes. The prevalence of rotator cuff tear increases with age (1), and is very common based on studies in symptomatic and asymptomatic populations. According to the literature reports, the prevalence of rotator cuff tears in cadaveric studies ranges from $5 \%$ to $40 \%$ of the population. There is a significant increase of prevalence in subjects over the age of $60(2,3)$.

The indications for surgical rotator cuff repair are full- and partial-thickness tears that involve $50 \%$ of the tendon thickness. Regardless of the high prevalence, there is still no consensus regarding the optimal treatment for rotator cuff tears. Numerous studies have been conducted to compare the different surgical approaches applied in rotator cuff tendon repair, in order to identify the optimal technique that provides the best function results and reduces the retear rates. With the remarkable development and evolution of surgical techniques and instruments, the surgical rotator cuff repair technique have transitioned from open to mini-open procedures, and finally to arthroscopic techniques (4). Arthroscopic repair of rotator cuff tear has been widely performed in recent years, with good outcomes reported in several studies (5-7).

Suture anchors are one of the essential requisites for successful arthroscopic rotator cuff repair. The firstgeneration suture anchor for arthroscopic rotator cuff repair is the metallic anchor. After clinical observation, the application of the metallic anchors has decreased due to the risk of complications. The most common complications are anchor loosening and migration, which can lead to cartilage damage and the failure of rotator cuff repair $(8,9)$. Bioabsorbable anchors were introduced as the secondgeneration suture anchors for arthroscopic rotator cuff repair to overcome these complications. The degradation and absorption of these bioabsorbable anchors has increased bone integration and reduced artifacts during postoperative MRI examination (10). However, bioabsorbable anchors have an unstable degeneration rate, which may not be appropriate with new bone formation (11).

With the development of suture anchor materials, polyether ether ketone (PEEK) has been introduced as a new suture anchor material with the advantage of being biologically inert and radiolucent. The PEEK suture anchor can also avoid the disadvantages of biocomposite anchors $(12,13)$. PEEK suture anchors have been designed with a variety of suitable anchor shapes and sizes for different patient applications. Due to its resistance to hydrolysis and oxidation, PEEK materials are very attractive for application in orthopedic surgery, which is considered to provide superior postoperative imaging and stable fixation benefits without the complications induced by the degradation of polymers $(14,15)$.

The Smith \& Nephew company has developed a variety of PEEK suture anchors that have been widely applied in arthroscopic rotator cuff repair. The clinical outcomes are promising and benefit patients significantly. However, the financial burden for patients is relatively high, especially in developing countries with poor insurance coverage. To reduce the costs of the treatment, Delta Medical has developed a series of PEEK suture anchors with a much lower cost, which have already been approved for the medical market by the National Medical Products Administration of China.

The purpose of this study was to compare the early clinical outcomes and MRI results of Delta Medical's PEEK suture anchor with those of Smith \& Nephew's PEEK suture anchor in patients with arthroscopic rotator cuff repair. The hypothesis is that the Delta Medical's PEEK suture anchor has a non-inferiority effect in patients with 
arthroscopic rotator cuff repair compared with those of Smith \& Nephew's PEEK suture anchor.

We present the following article in accordance with the CONSORT reporting checklist (available at https://dx.doi. org/10.21037/atm-21-6008).

\section{Methods}

\section{Sample size calculation}

In this study, we aimed to prove that the clinical effects of the Delta Medical's PEEK suture anchor products were not inferior to the similar Smith \& Nephew's PEEK suture anchors. The sample size was determined according to the non-inferiority test. The qualitative index (effective rate) was adopted as an evaluation index.

$$
n=n_{1}=n_{2}=\frac{2 \times\left(t_{a}+t_{\beta}\right)^{2} p(1-P)}{\delta^{2}}
$$

The significance level $\alpha$ value, power $1-\beta$, non-inferiority threshold $\delta$, and average total effective rate $\mathrm{P}$ value were determined according to the actual investigation of the efficacy of this type of product and the general statistical requirements. Based on the sample size calculation formula (as follows): $\mathrm{n}=\mathrm{n}_{1}=\mathrm{n}_{2}=48$ can be obtained.

Considering the possibility of cases falling off, the sample size of each group was set as 53 cases; that is, 53 cases in the experimental group and 53 cases in the control group.

$\mathrm{n}$ : estimated sample size; $\mathrm{n}_{1}$ and $\mathrm{n}_{2}$ are the sample sizes of the experimental and control groups, respectively.

$P$ : the average effective rate of the control group. Combined with the actual work experience of the main investigator, the average effective rate of the positive control substance $P$ was conservatively determined as $97 \%$.

$\delta$ : non-inferiority cut-off value. According to the "Guiding Principles for Selection of Non-inferiority Cut-off Value" issued by the European Drug Evaluation Organization (EMEA) (EMEA/CPMP/EWP/2158/99), and by the International Coordination Conference on Technical Requirements for Registration of Human Drugs (ICH)E9, E10 guidelines, a value of 0.1 was determined as the noninferiority threshold, which was close to the10\% (0.097) average effective rate of the control group.

$\alpha$ : significance level (false positive rate). According to "Biostatistics Technical Guidelines for Clinical Trials of Chemical Drugs and Biological Products", $\alpha$ was set as 0.05 on both sides.

$\beta: 1-\beta$ is the test efficiency (power). According to the
"Biostatistics Technical Guidelines for Clinical Trials of Chemical Drugs and Biological Products", $1-\beta$ was set as 0.8 .

\section{Study design and patient selection}

This study was a multicenter (Hunan Provincial People's Hospital, The First Affiliated Hospital of Hunan Normal University, People's Hospital of Inner Mongolia Autonomous Region, Zhongshan Traditional Chinese Medicine Hospital, The First Affiliated Hospital of Kunming Medical University), prospective, single-blind, randomized, controlled, two-parallel, clinical trial. A total of 106 consecutive patients who underwent arthroscopic rotator cuff repair between September 1, 2017 and March 21, 2019, were enrolled in four different medical centers and randomly divided into two groups. The allocation ratio is 1:1. Group 1 used Delta Medical's PEEK suture anchor for arthroscopic rotator cuff repair, while group 2 used Smith \& Nephew's PEEK suture anchor for arthroscopic rotator cuff repair.

Block randomization was performed by research assistants in different medical centers to allocate patients to one of the two treatment groups. An independent investigator, who was not involved in the surgical treatment, prepared and sealed opaque envelopes bearing the type of PEEK suture anchor used. The research assistants in different medical centers are responsible for patient enrollment. Following diagnostic arthroscopy, which was performed to confirm the lesion and the patient's eligibility for the study, the patients were randomized into one of the two treatment groups. Patients were not informed about which technique was used, either on the day of the surgery or at the follow-up visits. Also, the examiners who evaluated the patients' shoulders did not know the type of surgery performed.

Patients were enrolled in this study when a rotator cuff tear was diagnosed based on clinical examination and an MRI test. All procedures performed in this study involving human participants were in accordance with the Declaration of Helsinki (as revised in 2013). The study was approved by medical ethics board of Hunan Provincial People's Hospital, The First Affiliated Hospital of Hunan Normal University (No. 2017-05), People's Hospital of Inner Mongolia Autonomous Region (No. YWLCSYLL-2017-005-01), Zhongshan Traditional Chinese Medicine Hospital (No. 2017ZSZY-LL-003), and The First Affiliated Hospital of Kunming Medical University (No. 2017-QL-003), 

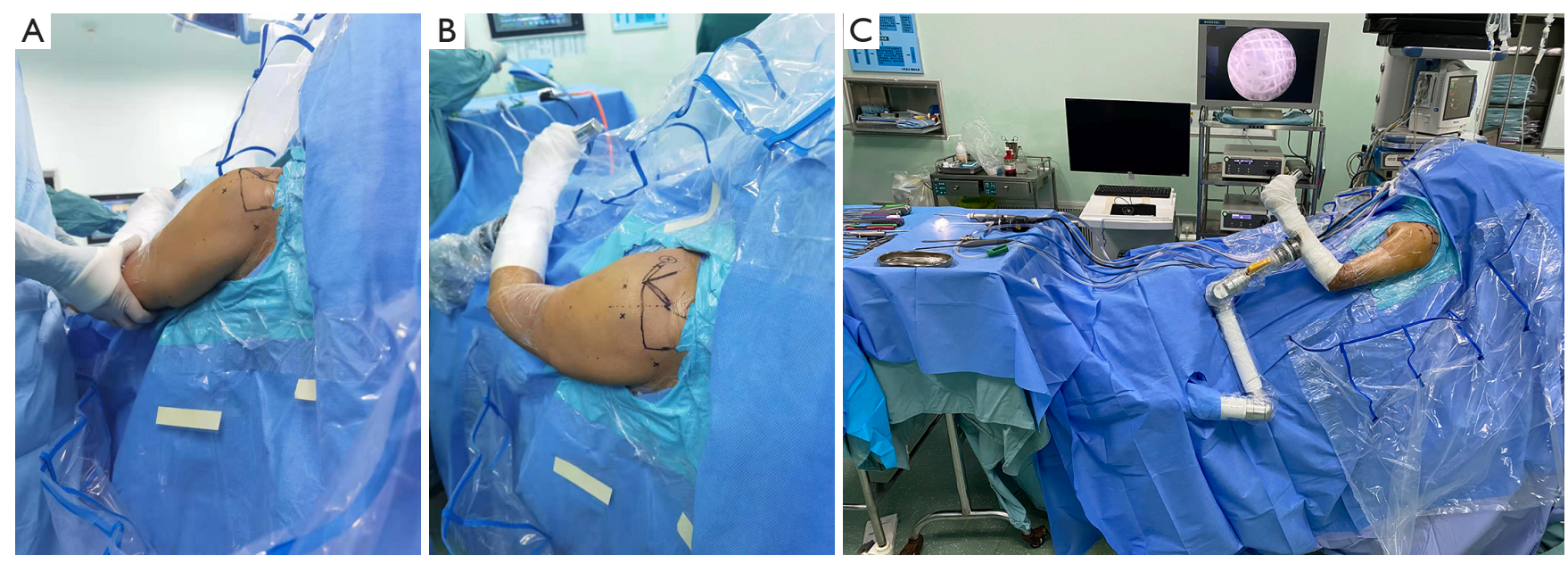

Figure 1 Portal marker and surgery position. (A) Lateral view; (B) top view; (C) overall view.

separately. Informed consent was taken from all the patients.

The inclusion criteria were as follows: (I) patients who were willing to be randomized to group 1 or group 2 and sign the informed consent form; (II) patients aged 18-75 years (including 18 and 75 years old), regardless of gender; (III) patients diagnosed with rotator cuff tear requiring arthroscopy surgery, without contraindications to suture anchor implantation; and (IV) patients with good compliance, willingness, and ability to conduct follow-up observation as required.

The exclusion criteria were as follows: (I) patients who had participated in other medical device trials within 3 months prior; (II) patients with severe allergies; (III) patients with abnormal liver and kidney function [SGPT (ALT) or SGOT (AST) or creatinine (CR)] 1.5 times the upper limit of normal; (IV) patients with coagulation dysfunction; (V) patients with serious heart and lung diseases that could restrict their participation in research and follow-up, or affect the scientific integrity of the research; (VI) patients with poor compliance, mental illness, mental disorders, and cooperation difficulties; (VII) patients with a positive pregnancy test, pregnant or breastfeeding women, those who had recently had a birth plan, and those who could take feasible contraceptive measures during the test period; (VIII) cases involving evidence that the subject is abusing drugs; (IX) patients with peripheral nerve injury at the surgical site; $(\mathrm{X})$ patients with myocardial infarction within 6 months and a history of cerebral infarction within 3 months; and (XI) patients with a history of cervical spondylotic myelopathy or radiculopathy.

\section{Arthroscopic technique}

Arthroscopic rotator cuff repair surgeries were performed by four senior orthopedic surgeons in the four different medical centers (i.e., the same senior orthopedic surgeon in each medical center). The surgeon who performed the surgery did not examine the patients preoperatively or postoperatively. General anesthesia was administered in all patients while in the beach chair position. Surgeries were performed with patients under general anesthesia in the beach chair position and the affected arm at approximately $45^{\circ}$ of abduction and $20^{\circ}$ of forward flexion (Figure 1). The distraction of the shoulder joint was accomplished with an adjustable traction arm. The conditions of the shoulder joint and level of rotator cuff tear lesion were visualized via a lateral port. Lesions of the rotator cuff tear were confirmed by arthroscopy from both the bursal and articular sides. Bleeding was controlled using radiofrequency and adrenalin mixed with the irrigation fluid. Once the rotator cuff tear had been defined, the edges of the tendon and footprint were judiciously debrided and repaired with either Delta Medical's PEEK suture anchor (group 1, 53 patients, Figure 2) or Smith \& Nephew's PEEK suture anchor (group 2, 53 patients, Figure 3). The number of PEEK suture anchors used varied with the size of the rotator cuff tear.

\section{Rebabilitation protocol}

The two groups received the same postoperative management and rehabilitation. In the first 6 weeks after 

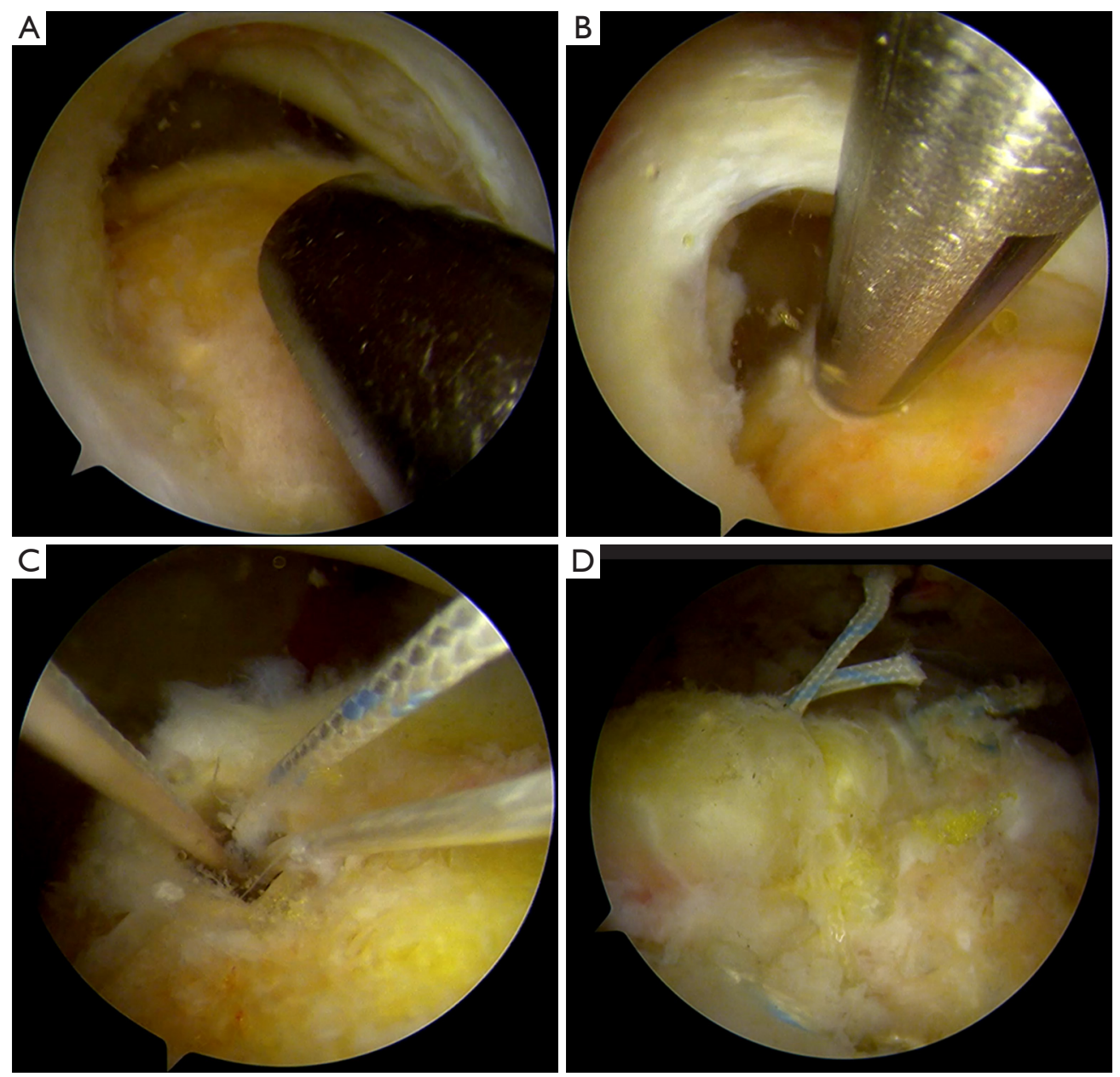

Figure 2 Final arthroscopic repair configuration after full-thickness supraspinatus tendon tear with Delta Medical's PEEK suture anchor. (A) Freshening the footprint area; (B) preparing the anchor insertion; (C) inserting the anchor; (D) final repair configuration. PEEK, polyether ether ketone.

surgery, all patients in the four medical centers used the same sling with an abduction pad for the affected arm. Active elbow flexion and extension, as well as passive external rotation, were allowed. However, terminal extension and overhead stretching were not allowed. The sling was removed after 6 weeks and isotonic strengthening of the affected muscles of the rotator cuff, deltoid muscle, and scapular muscles was initiated at 10-12 weeks postoperatively. This postoperative management and rehabilitation protocol was continued for 6 months in both groups. Heavy manual work and overhead activities were not allowed until after sufficient muscle strengthening at approximately 12 months after surgery.

\section{Clinical assessment}

Pre- and post-operative clinical assessments at each medical center were performed by one independent physical therapist blinded to the surgical repair technique performed. Assessments were performed on all patients preoperatively, and at 3 and 6 months postoperatively. The primary endpoint is 6 months post operation. A modified UCLA shoulder rating scale was used to evaluate strength (5 points), shoulder pain (10 points), function (10 points), active forward flexion (5 points), and patient satisfaction ( 5 points). The maximum obtainable score is 35 , and the results were classified as excellent (34-35 points), good (28-33 points), fair (21-27 points), or poor (0-20 points). 

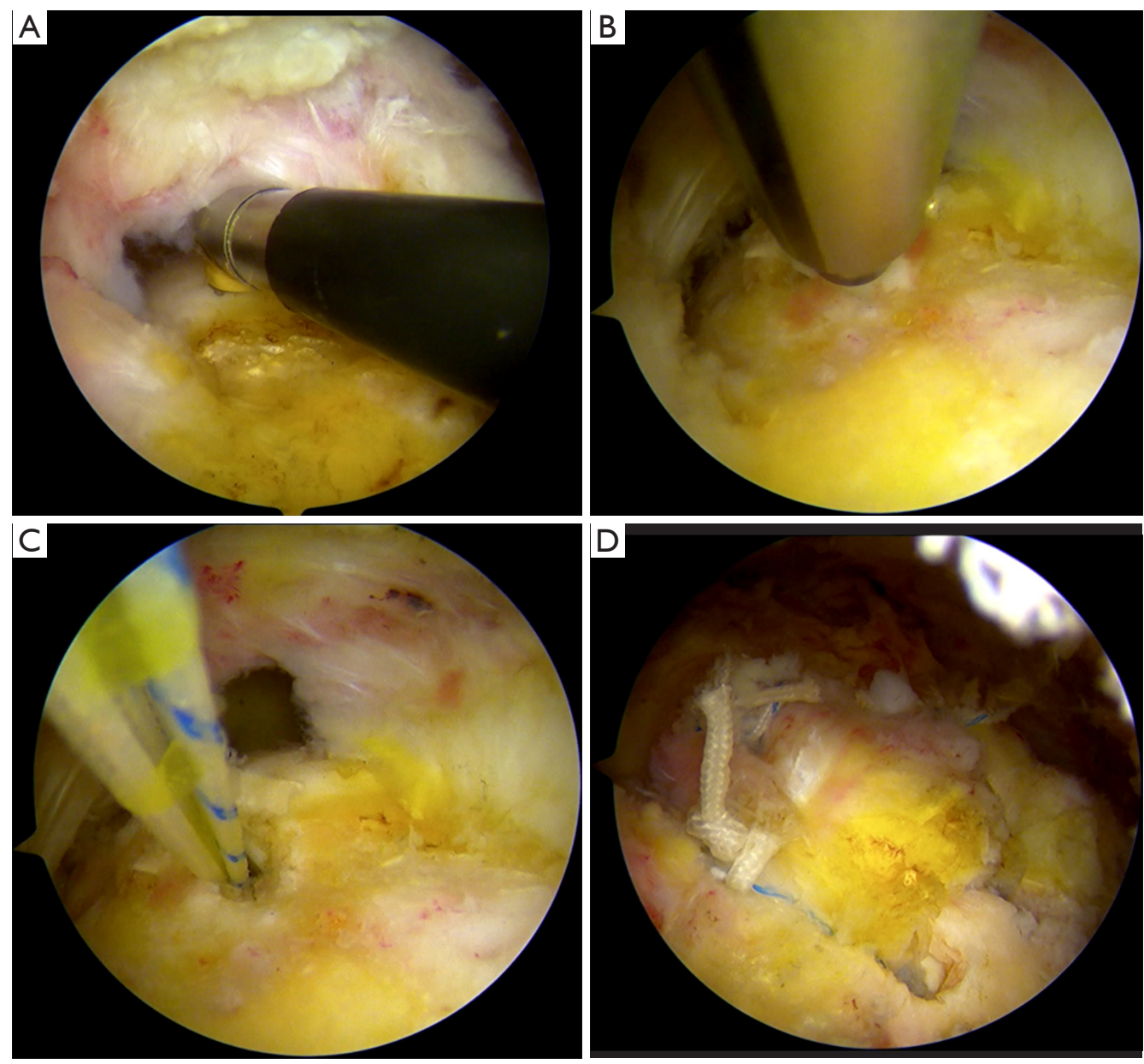

Figure 3 Final arthroscopic repair configuration after full-thickness supraspinatus tendon tear with Smith \& Nephew's PEEK suture anchor. (A) Freshening the footprint area; (B) preparing the anchor insertion; (C) inserting the anchor; (D) final repair configuration. PEEK, polyether ether ketone.

The clinical effective rate was calculated based on the UCLA shoulder rating scale results and classified as excellent and good at 6 months postoperatively.

MRI tests were performed on all patients at 3 and 6 months postoperatively to determine the healing process of the repaired tendon based on the Sugaya classification $(16,17)$. The repaired rotator cuff was classified as Sugaya I (repaired rotator cuff has complete continuity, normal thickness, and uniform signal in each layer), Sugaya II (repaired rotator cuff has complete continuity and normal thickness, with high signal areas visible locally), Sugaya III (the thickness of repaired rotator cuff is less than half of the normal rotator cuff, and there is no discontinuity, suggesting partial delamination), Sugaya IV (discontinuous signals can be seen in one to two layers in both the oblique coronal plane and the sagittal plane, suggesting a small fullthickness tear), and Sugaya V (large signal discontinuities above two levels can be seen in both the oblique coronal plane and the sagittal plane, suggesting a medium or large tear).

The pain visual analog scale (VAS) was applied to assess shoulder pain at rest, active activity, and passive activity on all patients preoperatively (at 3 months and 6 months). The results were classified as excellent (0 points, painless), good (1-3 points, mild pain, acceptable), fair (4-6 points, pain and affected sleep, tolerable), or poor (7-10 points, strong pain, affected sleep and appetite).

Clinical and functional range of motion and strength evaluations were performed on all patients preoperatively, and at 3 and 6 months postoperatively. Passive and active 
forward elevation (sagittal plane), internal and external rotation range of motion ( $\mathrm{ROM})\left(90^{\circ}\right.$ abduction) were measured and recorded using a standard universal goniometer. The active and passive abduction ranges of the patient's shoulder joint were evaluated. Both examiners performed three measurements for each clinical and functional range of motion and strength evaluations investigated. The average value for each variable was used for statistical analysis.

Computerized tomography (CT) was performed on all patients at 2 weeks and 3 months postoperatively to test the complications of PEEK suture anchor implantation, such as loosening, shifting, or breakage.

\section{Statistical analysis}

Data are presented as percentages for categorical variables, and as means and standard deviations for continuous variables. The statistical description was performed on demographic characteristics. Continuous variables were described using the mean, standard deviation, median, minimum, and maximum values. Categorical variables were described using frequencies. Baseline equilibrium statistical inferences were made on the demographic characteristics. Continuous variables used two independent samples $t$-test or Wilcoxon rank sum test according to the situation. Categorical variables used two independent samples chisquare test or Fisher exact probability method according to the situation. A per-protocol set (PPS) was used to conduct statistical analysis of the trial efficacy. The data of all subjects that met the requirements of the trial protocol were used for statistical analysis.

The UCLA scores were calculated in both groups preoperatively, and at 3 and 6 months after surgery, respectively. Two independent samples $t$-test or Wilcoxon rank sum test were used to analyze the differences between the two groups. According to the clinical evaluation results at 6 months postoperatively, the clinical effective rate was calculated, and the $95 \%$ confidence interval of the clinical effective rate difference between the two groups was finally calculated. The non-inferiority test was performed.

Concerning MRI examination, the VAS score, shoulder joint mobility measurement, or two independent samples t-test or Wilcoxon rank sum test was used for continuous variables, and two independent samples chi-square test or Fisher exact probability method were used for categorical variables, in order to compare the differences between the experimental and control groups.
An independent sample chi-square test (or exact probability method) was applied to analyze the CT examination results (whether loose, shifted, or broken) between two groups at each time point. Statistical significance was defined as $\mathrm{P}<0.05$.

\section{Results}

A total of 106 patients were enrolled in this study, and 104 patients completed the follow-up. The time period of recruitment is from September 1, 2017 and March 21, 2019. One patient was unwilling to continue participating in the study, and another was excluded after a postoperative review of the medical records, which found that they met the exclusion criteria 3 . To ensure the safety of the patient, we decided to conduct a complete follow-up of this patient (not included in the statistical analysis) (Figure 4). The mean age of the patients was 56.7 years (range, 29-74 years), and they all had a follow-up time of 6 months (except for the two who were lost to follow-up). The study population consisted of $64(61.5 \%)$ women and $40(38.5 \%)$ men. The mean bodyweight of the studied population was 61.5 $\mathrm{kg}$ (range, 36-99 kg), with a standard deviation of 10.52 (Table 1).

\section{UCLA scale}

The preoperative mean UCLA scores were 15.9 and 16.0 in groups 1 and 2, respectively, and there was no statistically significant difference between the two groups $(\mathrm{P}=0.285)$. In comparison with the preoperative status, both groups had a notable improvement in their UCLA scores at 3 and 6 months postoperatively. At 3 months, the mean UCLA scores in groups 1 and 2 were 25.0 and 25.1, respectively, and were 30.1 and 30.7 at 6 months postoperatively. Neither of the assessments of UCLA scores between the two groups at 3 and 6 months postoperatively showed statistically significant differences $(\mathrm{P}=0.885$ and 0.340 , respectively) (Table 2). Furthermore, pain, function, anterior flexion activity, anterior flexion strength, and satisfaction of UCLA scores scale, respectively, neither of these parameters showed statistically significant differences between the two groups throughout the entire follow-up period.

The clinical effective rate was calculated based on the UCLA shoulder rating scale results, and was classified as excellent and good at 6 months after surgery. Nine patients in group 1 did not achieve excellent or good UCLA scores, while in group 2, 13 patients did not obtain excellent or 


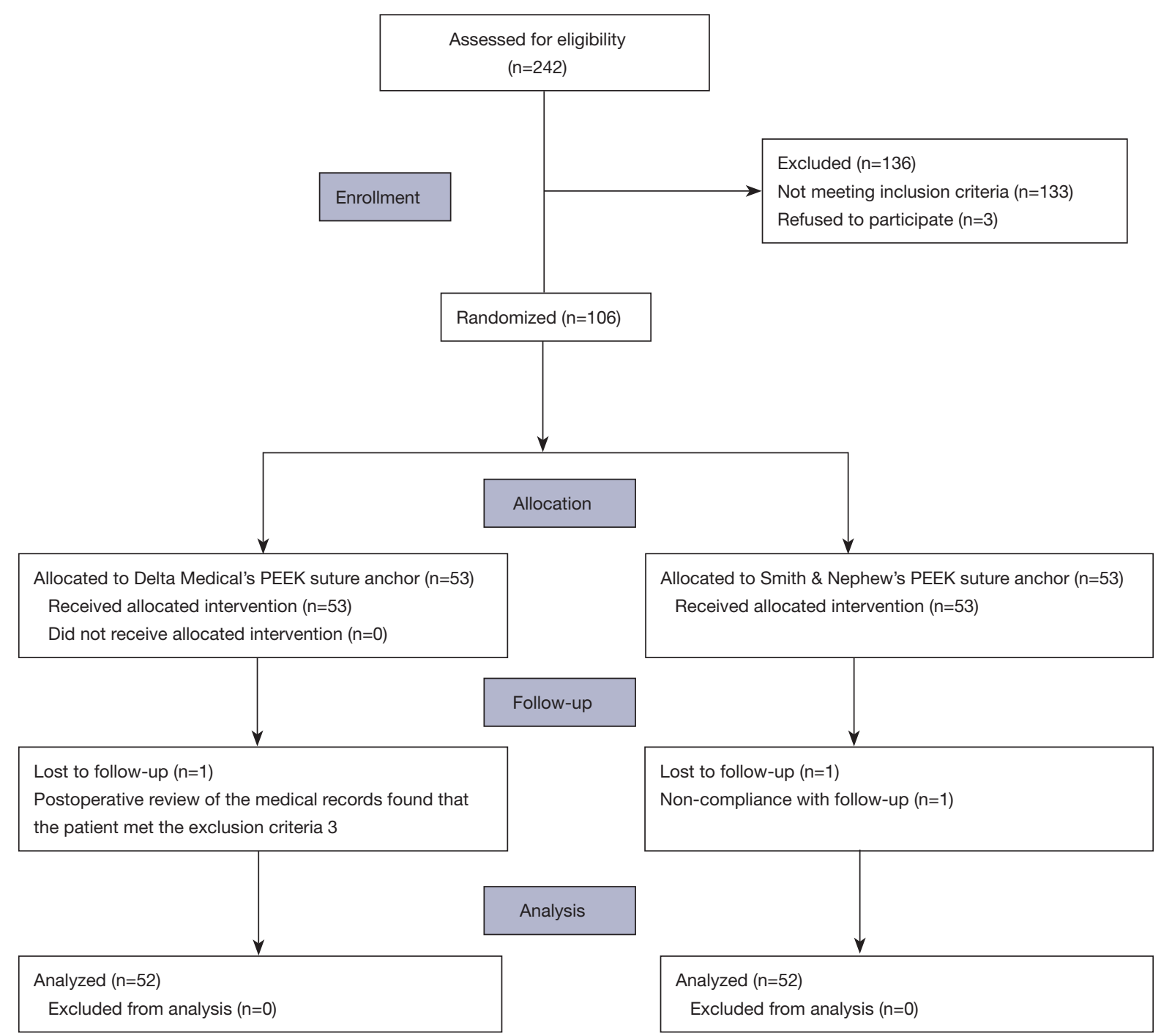

Figure 4 Patient selection flow chart.

good UCLA scores, which was significantly higher than group 1 . The clinical effective rate in group 1 was $82.7 \%$, compared with $75 \%$ in group 2 . However, the difference between the groups was not statistically significant (noninferiority analysis $\mathrm{P}=0.0132$ ). The $95 \%$ confidence interval was -0.0794 to 0.2332 (Table 3).

Rotator cuff repair integrity was assessed by MRI at 3 and 6 months postoperatively, and graded using the Sugaya classification (Figure 5). Dichotomizing the MRI results in terms of integrity and rupture (Sugaya classes I, II, III = intact; Sugaya classes IV and V = retorn), MRI scanning results showed that 49 patients in group 1 had an intact rotator cuff, compared with 48 patients in group 2 at 3 months postoperatively. Nine patients $(17.3 \%)$ in group 1 had Sugaya type I rotator cuff integrity at 3 months postoperatively, while at the same time, 14 patients (26.9\%) in group 2 had Sugaya type I rotator cuff integrity. At 6 months after surgery, $13(25.0 \%)$ patients had Sugaya type I rotator cuff integrity in group 1 was, compared to 11 $(21.2 \%)$ patients in group 2. After double-checking the MRI scanning results with the radiologist, an interesting finding was that the complete healing cases decreased from 14 ( 3 months) to 11 (6 months) in group 2. This may be due to extensive and inappropriate exercise. The fatty infiltration of the supraspinatus muscle did not increase significantly in the time between surgery and final follow-up evaluation in 
Table 1 Demographics data of the two groups

\begin{tabular}{lccc}
\hline Variables & Group 1 & Group 2 & P value \\
\hline Number of cases & 52 & 52 & 0.3828 \\
Median age (SD) & $55.9(9.81)$ & $57.5(7.99)$ & 0.2265 \\
Female/male & $23 / 29$ & $17 / 35$ & 0.4878 \\
Height (SD) & $1.62(0.070)$ & $1.61(0.069)$ & 0.8557 \\
Weight (SD) & $61.25(11.372)$ & $61.63(10.098)$ & \\
\hline
\end{tabular}

SD, standard deviation.

Table 2 Functional assessment: UCLA score

\begin{tabular}{llll}
\hline Groups & N & Mean (SD) & P value \\
\hline Preoperatively & 52 & $16.0(4.94)$ & 0.8915 \\
Group 1 & 52 & $15.9(5.09)$ & 0.8850 \\
Group 2 & & & $25.0(4.22)$ \\
3 months & 52 & $25.1(5.20)$ & 0.3400 \\
Group 1 & 52 & $30.1(3.47)$ & $30.7(2.83)$ \\
Group 2 & & 52 & \\
months & 52 & & \\
Group 1 & 52 & \\
\hline
\end{tabular}

UCLA, The University of California at Los Angeles; SD, standard deviation.

Table 3 Clinical effective rate ${ }^{\mathrm{a}}$

\begin{tabular}{|c|c|c|c|c|c|c|c|c|}
\hline Groups & $\begin{array}{l}\text { Excellent or } \\
\text { good }\end{array}$ & Rate & \multicolumn{2}{|c|}{ Difference test } & \multicolumn{2}{|c|}{ Non-inferiority analysis } & \multicolumn{2}{|c|}{$95 \% \mathrm{Cl}$} \\
\hline Group 1 & 43 & $82.7 \%$ & 0.9224 & 0.3368 & 2.2188 & 0.0132 & -0.0794 & 0.2332 \\
\hline Group 2 & 39 & $73.6 \%$ & & & & & & \\
\hline
\end{tabular}

a, this data is based on the UCLA score. UCLA, The University of California at Los Angeles; Cl, confidence interval.

both groups. Only one case of a complete retear occurred in group 1 , and this difference was not statistically significant (Table 4).

\section{VAS score}

Postoperatively, the mean VAS scores at rest were 3.6 and 4.3 in groups 1 and 2, respectively. Under active activity, the preoperative mean VAS scores were 5.6 and 6.0 in groups 1 and 2, respectively. Furthermore, the preoperative mean VAS scores under passive activity were 5.9 and 6.3 in groups 1 and 2, respectively. Neither of these preoperative mean VAS scores showed statistically significant differences between the two groups. Overall, patients experienced significant pain relief after rotator cuff repair in both groups. The assessment of mean VAS scores at 3 and 6 months after surgery showed a significant decrease in both groups $(\mathrm{P}<0.01)$. Compared with group 2 , the mean VAS scores in group 1 did not reveal statistically significant differences at 3 and 6 months after surgery. At 3 months postoperatively, the mean VAS scores at rest were 1.6 and 1.7 in groups 1 and 2, respectively $(\mathrm{P}=0.887)$, while at 6 months 

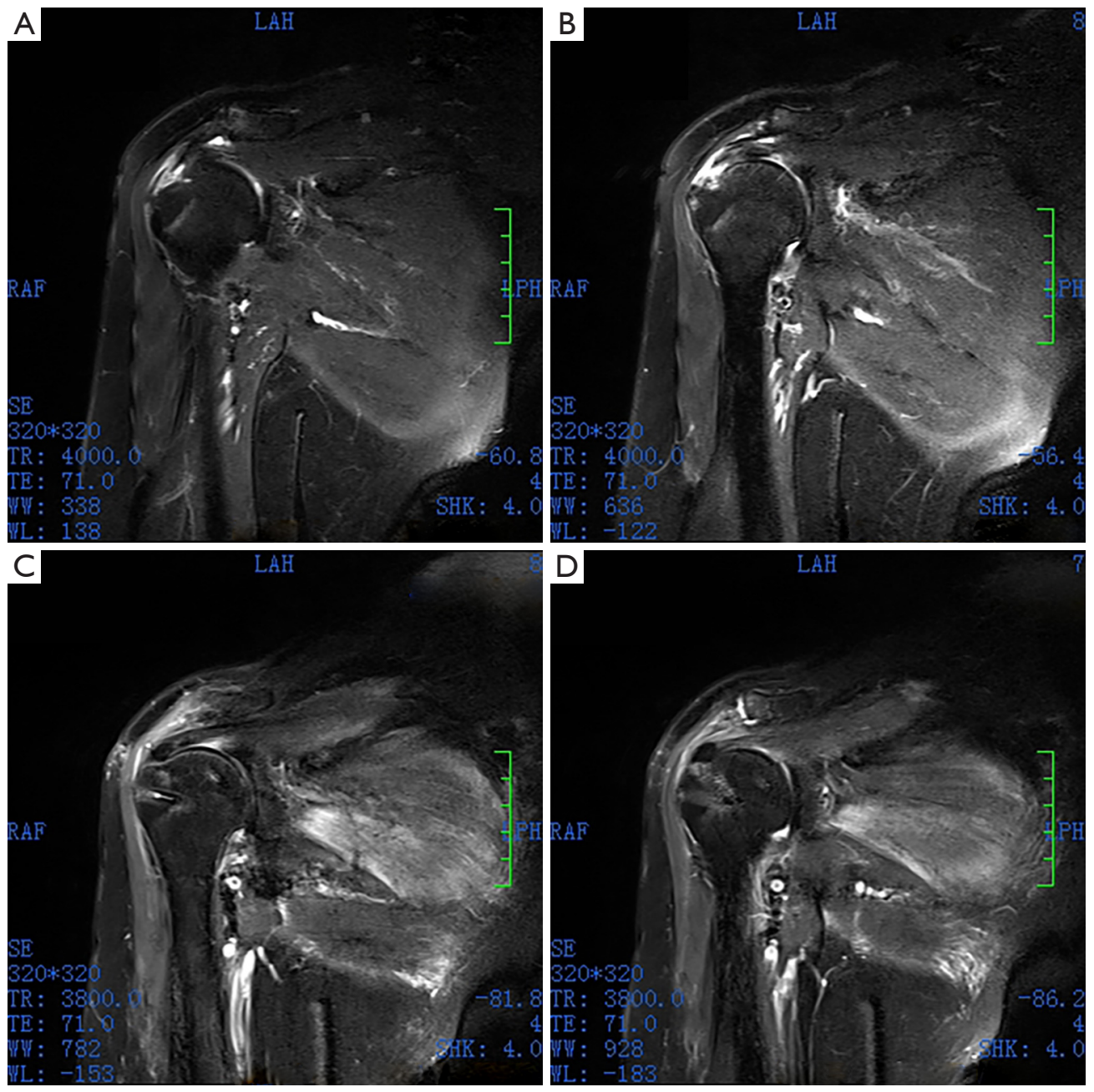

Figure 5 Coronal MRI of the supraspinatus tendon before and after arthroscopic rotator cuff repair. (A) Preoperative MRI of patient in Smith \& Nephew's PEEK suture anchor group. Freshening the footprint area; (B) postoperative MRI of patient in Smith \& Nephew's PEEK suture anchor group; (C) preoperative MRI of patient in Delta Medical's PEEK suture anchor group; (D) postoperative MRI of patient in Delta Medical's PEEK suture anchor group. MRI, magnetic resonance imaging.

postoperatively, these scores were 0.6 and 0.8 in groups 1 and 2, respectively. The mean VAS scores under active and passive activity exhibited similar results. The corresponding data are described in Table 5.

\section{Shoulder joint mobility measurement}

The active range of motion (anteflexion, abduction) increased significantly in both groups after rotator cuff repair. In group 1, the mean forward anterior flexion improved from 150.2 preoperatively to 119 at 6 months postoperatively $(\mathrm{P}<0.01)$, while the mean abduction range of motion improved from 103.1 preoperatively to 135.3 at
6 months postoperatively $(\mathrm{P}<0.01)$. In group 2 , mean forward anterior flexion improved from 132.3 preoperatively to 160.1 at 6 months postoperatively $(\mathrm{P}<0.01)$, while the mean abduction range of motion improved from 109.3 preoperatively to 137.6 at 6 months postoperatively $(\mathrm{P}<0.01)$. No significant differences in the range of motion were found at each follow-up time-point between the two groups $(\mathrm{P}>0.01)$.

Meanwhile, there were no significant changes in the extorsion range of motion (active and passive) in both groups after surgery. The mean extorsion range of motion in group 1 was 41.2 before surgery, compared with 46.9 at 6 months after surgery $(\mathrm{P}>0.01)$. The mean extorsion range 
Table 4 Characteristics of the repaired tendon according to the Sugaya classification ${ }^{\mathrm{a}}$

\begin{tabular}{|c|c|c|c|c|}
\hline Postoperatively & Type & Group 1 & Group 2 & $P$ value \\
\hline \multirow{3}{*}{3 months } & Type II & 33 (63.5\%) & 31 (59.6\%) & \\
\hline & Type III & 7 (13.5\%) & $4(7.7 \%)$ & \\
\hline & Type IV & $2(3.8 \%)$ & $3(5.8 \%)$ & \\
\hline \multirow[t]{4}{*}{6 months } & Type I & $13(25.0 \%)$ & $11(21.2 \%)$ & 0.1559 \\
\hline & Type II & $24(46.2 \%)$ & $31(59.6 \%)$ & \\
\hline & Type III & $10(19.2 \%)$ & 3 (5.8\%) & \\
\hline & Type IV & $4(7.7 \%)$ & 7 (13.5\%) & \\
\hline
\end{tabular}

a, the data comes from the Sugaya classification of the shoulder joint. Subjects with repeated evaluations keep records with higher Sugaya classification. The $\mathrm{P}$ value was derived from the chi-square test.

Table 5 Pain level assessment: VAS score

\begin{tabular}{|c|c|c|c|c|c|c|c|c|c|}
\hline Variable & \multicolumn{3}{|c|}{ Preoperatively } & \multicolumn{3}{|c|}{3 months } & \multicolumn{3}{|c|}{6 months } \\
\hline At rest & $3.6(2.24)$ & $4.2(2.93)$ & 0.2203 & $1.6(1.86)$ & 1.7 (1.94) & 0.7976 & $0.6(0.97)$ & $0.8(1.26)$ & 0.4344 \\
\hline Active activity & $5.6(1.89)$ & $6.0(2.00)$ & 0.3947 & $3.1(1.67)$ & $3.3(2.32)$ & 0.6304 & $1.5(1.45)$ & $1.5(1.46)$ & 1.0000 \\
\hline Passive activity & $5.9(2.18)$ & $6.3(2.04)$ & 0.3102 & $3.2(1.89)$ & $4.0(2.30)$ & 0.0599 & $1.6(1.63)$ & $1.7(1.65)$ & 0.6762 \\
\hline
\end{tabular}

Data are shown as mean (SD). VAS, visual analog scale.

Table 6 Shoulder joint mobility measurement

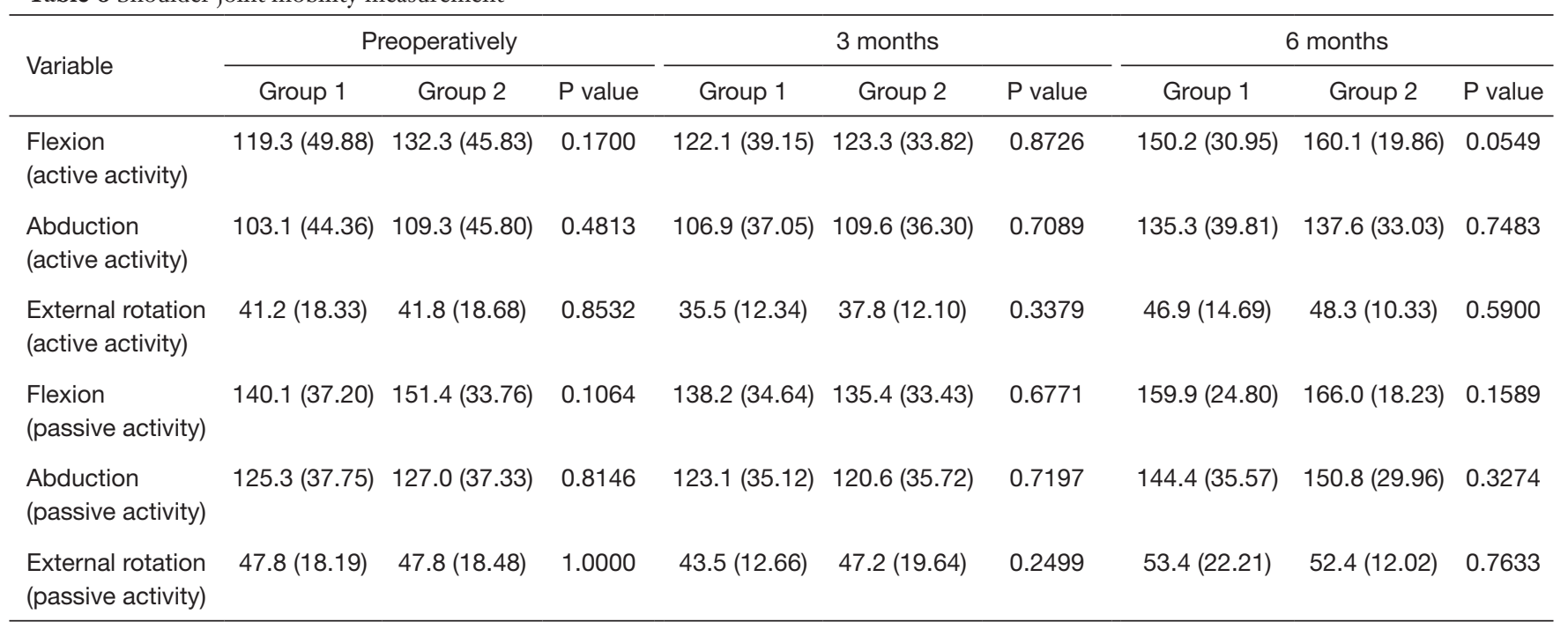

Data are shown as mean (SD). SD, standard deviation. 
Table 7 Implant-related complications based on CT examination

\begin{tabular}{|c|c|c|c|c|c|c|}
\hline Variable & \multicolumn{3}{|c|}{2 weeks } & \multicolumn{3}{|c|}{3 months } \\
\hline Loosening & 0 & 1 & 0.3150 & 1 & 0 & 0.3150 \\
\hline Shifting & 0 & 0 & & 0 & 0 & \\
\hline Breakage & 0 & 0 & & 0 & 1 & 0.3150 \\
\hline
\end{tabular}

of motion in group 2 was 41.8 before surgery, compared with 48.3 at 6 months after surgery $(\mathrm{P}>0.01)$ (Table 6$)$.

\section{CT examination results}

CT was performed on all patients at 2 weeks and 3 months postoperatively to test the complications of PEEK suture anchor implantation, such as loosening, shifting, or breakage. Based on the results, there was only one patient in group 2 who had an anchor loosening at 2 weeks postoperatively, and one patient in group 1 who had an anchor loosening at 3 months postoperatively. No shifting or breakage of anchor occurred in both groups at 2 weeks and 3 months postoperatively (Table 7).

\section{Complications}

There were no major intra- or post-operative complications, such as infection, and vessel or nerve injury. There were two patients in group 1 and three patients in group 2 who had stiffness in their shoulders after surgery. All stiffness in both groups improved significantly after active function exercise.

\section{Discussion}

The arthroscope was first introduced to treat rotator cuff diseases of impingement syndrome. Several studies have reported the treatment of subacromial impingement syndrome using arthroscopic subacromial decompression in patients with positive impingement signs (18). When performing arthroscopic rotator cuff repair, we should bear in mind the indications for surgery. The concept of chronic and traumatic cuff tear should be recognized and the acromion-humeral distance (AHD) needs to be taken into consideration for appropriate surgical indication (19). When treating partial rotator cuff tear, trans-tendon repair seems to have the same favorable clinical results as cuff take-down and full-thickness repair (20). There is still controversy regarding the treatment of the long head of the biceps tendon during arthroscopic rotator cuff repair. A randomized, controlled study showed that there was no significant difference in treatment between tenotomy and tenodesis of the long head of the biceps tendon. This suggested that we could perform tenotomy in order to shorten the surgery time and reduce pain (21). With the development of arthroscopic repair techniques for rotator cuff tears, many researchers have emphasized the application of double-row repair, hoping to add strength to the repaired tendon and decrease the anatomic failure rate $(17,22,23)$. However, the improvement with double-row repair compared with the single-row repair is still debatable; there are still no animal or clinical studies that have clearly demonstrated any improvement in the results when using double-row repair $(24,25)$.

Suture anchor materials with improved biocompatibility and reduced related complications were introduced in recent years. Biocomposite suture anchors with improved biocompatibility by regulating the properties of polymers and supplementing osteoconductive materials reduced the extent of cyst formation around the suture anchors (26). However, the new bone ingrowth sometimes fails to meet with the process of the degradation of biodegradable suture anchor materials. In a study of a biodegradable screw with $\beta$-tricalcium phosphate-poly-Lactic acid, the new bone ingrowth only $10 \%$ of the bone tunnel at 50 months postsurgery (27). Biocomposite suture anchors with ceramic compounds composed of metallic and non-metallic elements possess the features of toughness and brittleness, which limit their further application (11). PEEK was introduced as a new suture anchor material with the advantage of being biologically inert and radiolucent, and overcame these disadvantages of biocomposite anchors (28). PEEK is a hydrophobic polymer with a similar elasticity modulus to cortical bone. PEEK implants have shown advantages of high load, as well as reduced inflammatory reactions and cytotoxicity $(13,29)$. PEEK suture anchors are considered to provide stable fixation and superior postoperative imaging (30). There was a prospective randomized 
clinical trial compared the open-construct PEEK suture anchor with non-vented bio-composite suture anchor in arthroscopic rotator cuff repair. The results showed that the shoulder function was improved after complete rotator cuff repair and similar clinical outcomes were achieved regardless of suture anchor material and shape. However, the open-construct PEEK anchor provided better bone ingrowth into the anchor than the non-vented biocomposite anchor at 6 months after arthroscopic rotator cuff repair (28). Therefore, the PEEK suture anchor is now widely applied in arthroscopic rotator cuff repair.

In our study, we investigated the PEEK suture anchor developed by Delta Medical in arthroscopic rotator cuff repair, and compared its clinical and MRI outcomes with those of Smith \& Nephew's PEEK suture anchor. A total of 106 patients were enrolled in this study and the results indicated that patients in arthroscopic rotator cuff repair using PEEK suture anchor developed either by Delta Medical or Smith \& Nephew had a significant improvement in clinical outcomes after surgery. Considering pain, function, anterior flexion activity, anterior flexion strength, and satisfaction of the UCLA scores scale, respectively, neither of these parameters showed statistically significant differences between the two groups throughout the followup time. The clinical effective rate was calculated based on UCLA results, and was classified as excellent and good at 6 months after surgery. There were nine patients in group $1(82.7 \%)$ and 13 patients in group $2(75 \%)$ who did not achieve excellent or good UCLA scores. However, the difference between the two groups was not statistically significant (non-inferiority analysis $\mathrm{P}=0.013$ ).

MRI was applied to assess the rotator cuff repair integrity postoperatively using the Sugaya classification. The results showed that at 3 months after surgery, 49 patients $(94.2 \%)$ in group 1 had intact rotator cuff, compared with 48 patients $(92.3 \%)$ in group 2 . At 6 months after surgery, $13(25.0 \%)$ patients in group 1 had Sugaya type I rotator cuff integrity, compared with $11(21.2 \%)$ patients in group 2. There were no significant differences between the two groups.

The patients experienced significant pain relief after rotator cuff repair in both groups and a marked decline in the VAS scores was observed in both groups after rotator cuff repair surgery. Compared with group 2, the mean VAS scores in group 1 did not exhibit statistically significant differences at 3 and 6 months postoperatively. Active range of motion (anteflexion, abduction) increased notably in both groups after rotator cuff repair. Similarly, no significant differences in the range of motion were observed at each follow-up time-point between the two groups. Meanwhile, there were no significant changes in the extorsion range of motion (active and passive) in both groups after surgery. The mean extorsion range of motion in group 1 before surgery was 41.2 , compared with 46.9 at 6 months after surgery $(\mathrm{P}>0.01)$, while in group 2 , this was 41.8 preoperatively, compared with 48.3 at 6 months postoperatively $(\mathrm{P}>0.01)$.

Based on the CT examination results, there was only one patient in group 2 who had an anchor loosening at 2 weeks postoperatively, and there was one patient in group 1 who had an anchor loosening at 3 months postoperatively. No shifting or breakage of anchor occurred in either group at 2 weeks and 3 months postoperatively. There were no major intra- or post-operative complications, such as infection, and vessel or nerve injury.

This multicenter, prospective, single-blind, randomized, controlled, clinical trial revealed that rotator cuff tear patients repaired using PEEK suture anchor developed either by Delta Medical or Smith \& Nephew under arthroscopy had a significant improvement in clinical outcomes after surgery. When comparing Delta Medical's PEEK suture anchor with Smith \& Nephew's PEEK suture anchor, no significant differences were observed in terms of UCLA scores, VAS scores, MRI, and range of motion. This indicates that Delta Medical's PEEK suture anchor has a non-inferiority effect compared with Smith \& Nephew's PEEK suture anchor. This is important considering that the financial burden for patients using Smith \& Nephew's PEEK suture anchor is relatively high, especially in developing countries with poor insurance coverage. Delta Medical's products with a non-inferiority effect and much lower cost, which have already been approved for the medical market by the National Medical Products Administration of China, may be a suitable alternative for rotator cuff tear patients, especially those with lower incomes. The limitation of this trial is that the followup time was relative short, and a longer follow-up time is needed to verify the long-term effect of Delta Medical's PEEK suture anchor.

\section{Conclusions}

Delta Medical's PEEK suture anchor has a non-inferiority clinical effect compared with Smith \& Nephew's PEEK suture anchor in rotator cuff tear patients under arthroscopy surgery. The lower cost of Delta Medical's PEEK suture 
anchor may provide an alternative for rotator cuff tear patients.

\section{Acknowledgments}

Funding: This work was supported, in part, by the Scientific Research Project of Hunan Provincial Health Special Fund (A2020-02, P Gao); the Hunan Province Technological Innovation Guidance Plan-Clinical Medical Technology Innovation Guidance Project (2018SK50725, HT Wang); and the Key R\&D Program of Hunan Province (2020SK2117, J Wang).

\section{Footnote}

Reporting Checklist: The authors have completed the CONSORT reporting checklist. Available at https://dx.doi. org/10.21037/atm-21-6008

Trial Protocol: Available at https://dx.doi.org/10.21037/atm21-6008

Data Sharing Statement: Available at https://dx.doi. org/10.21037/atm-21-6008

Conflicts of Interest: All authors have completed the ICMJE uniform disclosure form (available at https://dx.doi. org/10.21037/atm-21-6008). The authors have no conflicts of interest to declare.

Ethical Statement: The authors are accountable for all aspects of the work in ensuring that questions related to the accuracy or integrity of any part of the work are appropriately investigated and resolved. All procedures performed in this study involving human participants were in accordance with the Declaration of Helsinki (as revised in 2013). The study was approved by medical ethics board of Hunan Provincial People's Hospital, The First Affiliated Hospital of Hunan Normal University (No. 2017-05), People's Hospital of Inner Mongolia Autonomous Region (No. YWLCSYLL-2017-005-01), Zhongshan Traditional Chinese Medicine Hospital (No. 2017ZSZY-LL-003), The First Affiliated Hospital of Kunming Medical University (No. 2017-QL-003), separately. Informed consent was taken from all the patients.

Open Access Statement: This is an Open Access article distributed in accordance with the Creative Commons
Attribution-NonCommercial-NoDerivs 4.0 International License (CC BY-NC-ND 4.0), which permits the noncommercial replication and distribution of the article with the strict proviso that no changes or edits are made and the original work is properly cited (including links to both the formal publication through the relevant DOI and the license). See: https://creativecommons.org/licenses/by-nc-nd/4.0/.

\section{References}

1. Randelli P, Stoppani CA, Zaolino C, et al. Advantages of Arthroscopic Rotator Cuff Repair With a Transosseous Suture Technique: A Prospective Randomized Controlled Trial. Am J Sports Med 2017;45:2000-9.

2. Tashjian RZ. Epidemiology, natural history, and indications for treatment of rotator cuff tears. Clin Sports Med 2012;31:589-604.

3. Tocci SL, Tashjian RZ, Leventhal E, et al. Biomechanical comparison of single-row arthroscopic rotator cuff repair technique versus transosseous repair technique. J Shoulder Elbow Surg 2008;17:808-14.

4. Warner JJ, Tétreault P, Lehtinen J, et al. Arthroscopic versus mini-open rotator cuff repair: a cohort comparison study. Arthroscopy 2005;21:328-32.

5. Liem D, Bartl C, Lichtenberg S, et al. Clinical outcome and tendon integrity of arthroscopic versus mini-open supraspinatus tendon repair: a magnetic resonance imaging-controlled matched-pair analysis. Arthroscopy 2007;23:514-21.

6. Ma HL, Chiang ER, Wu HT, et al. Clinical outcome and imaging of arthroscopic single-row and double-row rotator cuff repair: a prospective randomized trial. Arthroscopy 2012;28:16-24.

7. Spennacchio P, Banfi G, Cucchi D, et al. Long-term outcome after arthroscopic rotator cuff treatment. Knee Surg Sports Traumatol Arthrosc 2015;23:523-9.

8. Jeong JH, Shin SJ. Arthroscopic removal of proud metallic suture anchors after Bankart repair. Arch Orthop Trauma Surg 2009;129:1109-15.

9. Ozbaydar M, Elhassan B, Warner JJ. The use of anchors in shoulder surgery: a shift from metallic to bioabsorbable anchors. Arthroscopy 2007;23:1124-6.

10. Barber FA, Snyder SJ, Abrams JS, et al. Arthroscopic Bankart reconstruction with a bioabsorbable anchor. J Shoulder Elbow Surg 2003;12:535-8.

11. Dhawan A, Ghodadra N, Karas V, et al. Complications of bioabsorbable suture anchors in the shoulder. Am J Sports Med 2012;40:1424-30. 
12. Kuhn JE, Dunn WR, Sanders R, et al. Effectiveness of physical therapy in treating atraumatic full-thickness rotator cuff tears: a multicenter prospective cohort study. J Shoulder Elbow Surg 2013;22:1371-9.

13. Kurtz SM, Devine JN. PEEK biomaterials in trauma, orthopedic, and spinal implants. Biomaterials 2007;28:4845-69.

14. Barber FA, Herbert MA. All-Suture Anchors: Biomechanical Analysis of Pullout Strength, Displacement, and Failure Mode. Arthroscopy 2017;33:1113-21.

15. Barber FA, Herbert MA, Beavis RC, et al. Suture anchor materials, eyelets, and designs: update 2008. Arthroscopy 2008;24:859-67.

16. Skaliczki G, Paladini P, Merolla G, et al. Early anchor displacement after arthroscopic rotator cuff repair. Int Orthop 2015;39:915-20.

17. Sugaya H, Maeda K, Matsuki K, et al. Functional and structural outcome after arthroscopic full-thickness rotator cuff repair: single-row versus dual-row fixation. Arthroscopy 2005;21:1307-16.

18. Kappe T, Knappe K, Elsharkawi M, et al. Predictive value of preoperative clinical examination for subacromial decompression in impingement syndrome. Knee Surg Sports Traumatol Arthrosc 2015;23:443-8.

19. Thès A, Hardy P, Bak K. Decision-making in massive rotator cuff tear. Knee Surg Sports Traumatol Arthrosc 2015;23:449-59.

20. Castagna A, Borroni M, Garofalo R, et al. Deep partial rotator cuff tear: transtendon repair or tear completion and repair? A randomized clinical trial. Knee Surg Sports Traumatol Arthrosc 2015;23:460-3.

21. Zhang Q, Zhou J, Ge H, et al. Tenotomy or tenodesis for long head biceps lesions in shoulders with reparable rotator cuff tears: a prospective randomised trial. Knee Surg Sports Traumatol Arthrosc 2015;23:464-9.

22. Lafosse L, Brozska R, Toussaint B, et al. The outcome and structural integrity of arthroscopic rotator cuff repair with use of the double-row suture anchor technique. J Bone

Cite this article as: Gao P, Wang H, Xu Y, Li Y, Cai G, Wu Y, Huang Z, Li Q, Wang J. A non-inferiority comparison of Delta Medical's PEEK Suture Anchor and Smith \& Nephew's PEEK Suture Anchor in arthroscopic rotator cuff repair: a multicenter prospective single-blind randomized controlled clinical trial. Ann Transl Med 2021;9(24):1767. doi: 10.21037/atm-21-6008
Joint Surg Am 2007;89:1533-41.

23. Sugaya H, Maeda K, Matsuki K, et al. Repair integrity and functional outcome after arthroscopic double-row rotator cuff repair. A prospective outcome study. J Bone Joint Surg Am 2007;89:953-60.

24. Boyer P, Bouthors C, Delcourt T, et al. Arthroscopic double-row cuff repair with suture-bridging: a structural and functional comparison of two techniques. Knee Surg Sports Traumatol Arthrosc 2015;23:478-86.

25. Liem D, Dedy NJ, Hauschild G, et al. In vivo blood flow after rotator cuff reconstruction in a sheep model: comparison of single versus double row. Knee Surg Sports Traumatol Arthrosc 2015;23:470-7.

26. Kim SH, Kim DY, Kwon JE, et al. Perianchor Cyst Formation Around Biocomposite Biodegradable Suture Anchors After Rotator Cuff Repair. Am J Sports Med 2015;43:2907-12.

27. Barber FA, Dockery WD. Long-term absorption of betatricalcium phosphate poly-L-lactic acid interference screws. Arthroscopy 2008;24:441-7.

28. Kim JH, Kim YS, Park I, et al. A Comparison of OpenConstruct PEEK Suture Anchor and Non-Vented Biocomposite Suture Anchor in Arthroscopic Rotator Cuff Repair: A Prospective Randomized Clinical Trial. Arthroscopy 2020;36:389-96.

29. Güleçyüz MF, Mazur A, Schröder C, et al. Influence of Temperature on the Biomechanical Stability of Titanium, PEEK, Poly-L-Lactic Acid, and $\beta$-Tricalcium Phosphate Poly-L-Lactic Acid Suture Anchors Tested on Human Humeri In Vitro in a Wet Environment. Arthroscopy 2015;31:1134-41.

30. Seaman S, Kerezoudis P, Bydon M, et al. Titanium vs. polyetheretherketone (PEEK) interbody fusion: Metaanalysis and review of the literature. J Clin Neurosci 2017;44:23-9.

(English Language Editor: A. Kassem) 